\title{
Multidrug residues and antimicrobial resistance patterns in waste milk from dairy farms in Central California
}

\author{
P. N. Tempini, ${ }^{*}$ S. S. Aly, ${ }^{\star} †$ B. M. Karle, $\ddagger$ and R. V. Pereira* \\ *Department of Population Health and Reproduction, School of Veterinary Medicine, University of California, Davis, Davis 95616 \\ †Veterinary Medicine Teaching and Research Center, School of Veterinary Medicine, University of California, Davis, Tulare 93274 \\ $\ddagger$ Cooperative Extension, Division of Agriculture and Natural Resources, University of California, Orland 95963
}

\begin{abstract}
Waste milk (WM) is a common source of feed for preweaned calves in US dairy farms. However, limited information is available about characteristics of this product, including concentration of drug residues and potential hazards from antibiotic-resistant bacteria present in the milk. The aims of this cross-sectional study were to (1) identify and measure the concentration of antimicrobial residues in raw WM samples on dairy farms in the Central Valley of California, (2) survey farm management practices for factors associated with the occurrence of specific antimicrobial residues in raw WM, (3) characterize the antimicrobial resistance patterns of E. coli cultured from raw WM samples, and (4) evaluate the potential association between WM quality parameter and risk of identifying drug residues in milk. A single raw bulk tank WM sample was collected from dairy farms located in California's Central Valley $(\mathrm{n}=25)$. A questionnaire was used to collect information about farm management practices. Waste milk samples were analyzed for a multidrug residue panel using liquid chromatography-tandem mass spectrometry. Bacteria were cultured and antimicrobial resistance was tested using standard techniques; milk quality parameters (fat, protein, lactose, solids-not-fat, somatic cell count, coliform count, and standard plate count) were also measured. Of the 25 samples collected, $15(60 \%)$ contained detectable concentrations of at least 1 antimicrobial. Of the drug residue-positive samples, $44 \%(11 / 25)$ and $16 \%(4 / 25)$ had detectable concentrations of $\beta$-lactams and tetracycline, respectively. The most prevalent drug residues were ceftiofur $(\mathrm{n}=7$, $28 \%)$, oxytetracycline $(\mathrm{n}=4,16 \%)$, and cephapirin ( $\mathrm{n}$ $=3,12 \%$ ). No significant associations were identified between farm characteristics or management practices
\end{abstract}

Received January 5, 2018.

Accepted May 23, 2018.

${ }^{1}$ Corresponding author: rvpereira@ucdavis.edu and presence of drug residues in WM. In this study, $20 \%$ of farms did not pasteurize WM before feeding to calves. Two of the 10 Escherichia coli isolated from WM samples were multidrug resistant. Streptococcus spp. (n $=21,84 \%$ ) was the most common genus cultured from WM samples, followed by Staphylococcus spp. $(\mathrm{n}=20$, $80 \%)$ and $E$. coli $(\mathrm{n}=10,40 \%)$. Mycoplasma spp. was cultured from $2 \mathrm{WM}$ samples $(\mathrm{n}=2,8 \%)$. The presence of drug residues in WM at concentrations that increase selection of resistant bacteria indicates the need for additional studies targeting on-farm milk treatments to degrade drug residues before feeding to calves. The presence of multidrug-resistant E. coli in WM urges the need for on-farm practices that reduce calf exposure to resistant bacteria, such as pasteurization.

Key words: waste milk, drug residue, ceftiofur, antibiotic resistance

\section{INTRODUCTION}

The use of antimicrobials in livestock production and its effect on the development of antimicrobial resistance are a current public health concern. Although prevention approaches can reduce disease and the need for use of antimicrobial drugs to treat animals, drug use in food animals is an important tool to help ensure animal health and welfare. Despite these benefits, there is considerable concern from public health, food safety, and regulatory stakeholders regarding the use of antimicrobials in food animals because of possible selection of antimicrobial-resistant bacteria (Oliver et al., 2011). The selection of resistant bacteria can especially occur when antimicrobials are administered to food animals at regimens outside of labeled dosage and duration (Maron et al., 2013).

In the dairy industry, most antimicrobial drugs used to treat cows result in the milk from these animals being withheld from sale because of the presence of drug residues above the tolerable concentration established by the US Food and Drug Administration (FDA). Milk from cows treated with drugs that require a milk with- 
drawal period and transitional milk (transitioning from colostrum to saleable milk) are considered nonsaleable for human consumption. One-third of dairy farms in the United States use waste milk (WM) to feed preweaned dairy calves (USDA, 2008a). A study examining the effects of feeding pasteurized WM to preweaned calves found that calves consuming pasteurized WM had increased weaning weights and decreased mortality rates compared with calves consuming milk replacer (Godden et al., 2005). Additionally, the total savings of feeding pasteurized WM was $\$ 0.69 /$ calf per day (Godden et al., 2005). However, milk from cows receiving antimicrobial treatment during lactation and colostrum from cows treated with antimicrobials at dry-off may contain concentrations of drug residues, and such milk could lead to increased fecal shedding of antimicrobialresistant bacteria when fed to preweaned dairy calves (Pereira et al., 2014a; Maynou et al., 2017a; Ricci et al., 2017). Not all dry cow treatments have zero withdrawal milk days (in addition to the minimum dry period length required). As an example, the commercial drug used for dry cow treatment containing cephapirin benzathine (ToMorrow, Boehringer Ingelheim, Ingelheim am Rhein, Germany) states on its label that milk from treated cows must not be used for food during the first $72 \mathrm{~h}$ after calving.

In a recent study conducted in New York State, $82 \%$ of WM fed to calves contained $\beta$-lactam drug residues, including ceftiofur, penicillin G, and ampicillin (Pereira et al., 2014b). A subsequent study evaluating the effect of ingestion of raw milk containing antimicrobial drugs found an increased prevalence of antimicrobial resistance in Escherichia coli isolated from the feces of dairy calves (Pereira et al., 2014a). Based on 16S RNA sequencing of fecal samples, feeding milk containing drug residues also affected the developing enteric microbiota of calves, resulting in a microbiota that discriminated at the genus level in their weekly microbial profile (Pereira et al., 2016). A follow-up evaluation using shotgun sequencing to evaluate the effects of feeding $\mathrm{WM}$ on the function of the microbiota revealed differences between treatment and control groups for genes linked to stress response, regulation and cell signaling, and nitrogen metabolism (Pereira et al., 2018). For example, calves in the treatment group had a greater abundance of microbial genes related to zinc-regulated enzymes, allowing this microbiota to adapt better to lower concentrations of zinc in feed. Calves in the control group had a greater abundance of microbial genes related to osmotic stress, which could translate into this microbiota being able to withstand greater osmotic pressure variation, such as that caused by feed with high concentration of sugars or salts. Changes in the microbiota functions could directly affect the resilience of the microbiota to adjust to changes such as variations in nutrient content or TS percentage in milk fed. To the author's knowledge, no studies have evaluated the effects of drug residues at concentrations observed in WM on the gastrointestinal epithelium.

Limited information is available on antimicrobial drug residues and resistant bacteria in WM fed to preweaned dairy calves. California is the leading dairy state in the United States, with 1.7 million lactating cows and contributing almost $20 \%$ of the country's milk supply in 2015 (Monson et al., 2015), so the California dairy industry is particularly poised to address this research need. Public awareness and increasing concerns about the development of antimicrobial resistance associated with the use of antimicrobials in livestock production and the possible spread of resistance to human medicine require field data to provide scientific information for stakeholders.

The aims and hypotheses for this study were as follows. First, we aimed to identify and measure the concentration of antimicrobial residues in raw WM samples on dairy farms in the Central Valley of California; our hypothesis was that more than $50 \%$ of dairy farms in California would have drug residues in bulk tank WM and that ceftiofur would be the most common drug residues detected in WM based on previous reports screening drug residues in WM (Pereira et al., 2014a; USDA, 2016). Second, we aimed to survey farm management practices for factors associated with the occurrence of specific antimicrobial residues in raw WM; our hypothesis was that use of specific antimicrobial drugs or management practices that affect the frequency of antimicrobial drug use would result in higher prevalence of specific drugs in the WM bulk tank. Third, we aimed to evaluate the association between WM quality parameters and identification of drug residues in milk; our hypothesis was that milk quality parameters could be associated with prevalence of drug residues. It was also an objective of the study to characterize the antimicrobial resistance patterns of $E$. coli cultured from raw WM samples. To our knowledge, this is the first study to focus on addressing all the aims and objectives on WM from dairy farms in California.

\section{MATERIALS AND METHODS}

\section{Study Population and Design}

Raw hospital WM samples from 25 dairy farms were collected from herds in the following counties in the Central Valley in California: Tulare $(\mathrm{n}=12)$, Kings $(\mathrm{n}=2)$, Fresno $(\mathrm{n}=2)$, Madera $(\mathrm{n}=1)$, Solano $(\mathrm{n}=$ $1)$, Placer $(\mathrm{n}=1)$, Glenn $(\mathrm{n}=5)$, and Butte $(\mathrm{n}=1)$. Samples were collected from August 2016 to February 
2017. Study farms were identified through collaboration with private practitioners in Kings, Fresno, Madera, and Tulare counties or conveniently selected based on their working relationship with the University of California, Davis School of Veterinary Medicine, including the Veterinary Medicine Teaching and Research Center in Tulare, California, and a University of California Agriculture and Natural Resources Cooperative Extension dairy advisor. As part of a cross-sectional study design, each participating farm was visited once between September 2016 and March 2017. During the farm visit, a WM sample was collected and a questionnaire was completed through an in-person interview with the farm owner or manager. Sample size was calculated using a simple random sampling approach, adjusting for a finite population (Thrusfield, 2005). An estimated sample size of 25 farms was obtained when the following parameters were offered to the sample size formula: target population of 1,300 dairy farms (CDFA, 2017), an expected prevalence of $82 \%$ (Pereira et al., 2014b), $90 \%$ confidence interval, and a $12.5 \%$ desired absolute precision.

The current study did not fall within the criteria that would require the University of California Institutional Animal Care and Use Committee protocol and was therefore exempted. The study was reviewed by the University of California, Davis Institutional Review Board and granted exemption status.

\section{Milk Sample Collection and Processing}

Approximately $650 \mathrm{~mL}$ of raw, pooled milk with no additives or preservatives was collected from the WM bulk tank and placed into sterile milk tubes. The contents of the tank were thoroughly mixed before sample collection. For dairies that milked fresh and treated cows separately, only the bulk tank for the treated cows was sampled. All samples were collected by the authors or by farm managers. On the farm, the milk sample was divided into two $200-\mathrm{mL}$ sterile containers and four $60-\mathrm{mL}$ sterile containers. Immediately after collection, the containers were stored on ice and transported to the nearest study laboratory (Pereira Lab, Davis, CA, or Aly Lab, Tulare, CA), where they were refrigerated at $4^{\circ} \mathrm{C}$. Samples were then transported or shipped overnight in cold packaging to Sierra Dairy Laboratory (Tulare, CA) for milk quality parameter determination and to Eurofins Central Analytical Laboratories (CAL; New Orleans, LA) for drug residue quantification using liquid chromatography-tandem MS (LC-MS/MS). The concentrations of the stock solutions used in the LC-MS/MS corresponded to the active drug compound, and the amounts weighed were adjusted to take into account purity and any counter- ions. Last, 1 aliquot of each WM sample was frozen at $-20^{\circ} \mathrm{C}$ for subsequent bacterial culture at the Milk Quality Laboratory (Veterinary Medicine Teaching and Research Center, Tulare, CA).

\section{Questionnaire}

To determine farm characteristics, management practices, and antimicrobial drug usage, data were collected from interviewees using a structured questionnaire with 18 closed-ended questions and options for comments. Interviews were conducted face to face, and the interviewee was provided a copy of the questionnaire to follow during the interview process, allowing them to read the questions being asked. Interviews were conducted during the milk sample collection visit with the exception of 1 dairy, where the manager collected the samples and stored them in a refrigerator overnight until pick-up the following day. The questionnaire addressed general information about farm characteristics, management practices, and antimicrobial drug use. The information from the questionnaire is available in Supplemental File S1 (https://doi.org/10 .3168/jds.2018-14398). Based on approaches used by the USDA National Animal Health Monitoring System (NAHMS) reports and expected number of animals in dairy farms in California, multiple-choice questions for dairy herd size and number of preweaned calves were formatted into categories (USDA, 2016; CDFA, 2017). Questions were based on information from NAHMS on diseases in dairy cattle that result in higher use of antimicrobial drug use (USDA, 2008b).

\section{Milk Quality Parameters}

An aliquot of all WM samples was sent to Sierra Dairy Laboratory (Tulare, CA) to measure the following milk quality parameters: fat, protein, lactose, SNF, SCC, coliform count, and SPC. Milk quality parameters were measured using standard procedures.

\section{Quantification of Drug Residues Using LC-MS/MS}

A simple SNAP test for the identification of $\beta$-lactam drug residues (SNAP Beta-Lactam ST test; Idexx Laboratories Inc., Westbroook, ME) was performed on all WM samples in the study laboratories. Identification and quantification of drug residues by LC-MS/MS were performed at Eurofins CAL using a protocol that can detect and quantify up to 27 drugs (26 antimicrobials and 1 nonsteroidal anti-inflammatory drug, 5-hydroxyflunixin). The protocol for LC-MS/MS at Eurofins CAL followed a modified laboratory information bulletin by the FDA (FDA, 2011a), which was modified and inter- 
nally validated by Eurofins CAL to include testing for ceftiofur. Briefly, the reagents and materials used for LC-MS/MS were deionized water $(18.2 \mathrm{M} \Omega / \mathrm{cm})$, highpurity chromatographic- and spectrophotometric-grade acetonitrile and methanol, and formic acid at $96 \%$ purity. The limits of quantification for the 27 drugs screened using LC-MS/MS are shown in Supplemental Table S1 (https://doi.org/10.3168/jds.2018-14398).

\section{Milk Cultures and Antimicrobial Susceptibility Test}

All microbiological testing was performed at the Milk Quality Laboratory (Veterinary Medicine Teaching and Research Center, Tulare, CA). Waste milk samples were thawed, plated by streaking $0.01 \mathrm{~mL}$ on bovine blood agar, and incubated at $37^{\circ} \mathrm{C}$ for $48 \mathrm{~h}$. Bacteria isolated on culture medium were identified according to colony morphology, hemolytic characteristics, Gram stain reaction, and catalase and coagulase tests. Estimation of number of viable bacteria at the genus level to detect the most common mastitis pathogens was performed using the serial dilution method. To detect Mycoplasma spp., WM samples were directly cultured on modified Hayflick agar as well as in an enriched Mycoplasmaspecific broth and incubated at $37^{\circ} \mathrm{C}$ in $4 \% \mathrm{CO}_{2}$ for 7 d. To discriminate between Acholeplasma spp. and Mycoplasma spp., a digitonin disk inhibition test was performed.

Escherichia coli colonies isolated from WM samples were tested for antimicrobial susceptibility using a Kirby Bauer disk diffusion agar assay in accordance with the guidelines published by the Clinical and Laboratory Standards Institute (CLSI, 2015). We chose to test E. coli isolates because it is a commonly used model for evaluating effects of antimicrobials on gram-negative bacteria, and several studies have examined the effect of feeding WM to calves on the occurrence of resistance in fecal E. coli (Pereira et al., 2014a; Maynou et al., 2017b). Antimicrobial susceptibility for all E. coli isolates was initially assessed using a standard panel used at the Milk Quality Laboratory for mastitis pathogens containing 8 antimicrobials (10 $\mu \mathrm{g}$ of ampicillin, 30 $\mu \mathrm{g}$ of cephalothin, $15 \mu \mathrm{g}$ of erythromycin, 10 units of penicillin, $2 \mu \mathrm{g}$ of pirlimycin, $30 \mu \mathrm{g}$ of tetracycline, 10 units/30 $\mu \mathrm{g}$ of penicillin/novobiocin, and $30 \mu \mathrm{g}$ of ceftiofur) to which a $250 \mu \mathrm{g}$ sulfisoxazole disk was added. Penicillin, penicillin/novobiocin, and pirlimycin were excluded from the analysis because of the weak spectrum of action against gram-negative bacteria, whereas erythromycin was excluded because of its previously reported weak activity against E. coli (Maynou et al., 2017b). Inclusion of these drugs in the analysis may have resulted in a false increase in the prevalence of antimicrobial resistance and would serve as an addi- tional source of bias. Susceptibility of the isolates to antimicrobial drugs was categorized as susceptible, intermediate, or resistant by measuring the inhibition zone according to interpretive criteria and breakpoints established by CLSI guidelines (CLSI, 2013, 2015; Supplemental Table S2, https://doi.org/10.3168/jds .2018-14398). Multidrug-resistant isolates were defined as having resistance to 3 or more classes of antimicrobials (Pereira et al., 2014a).

\section{Statistical Analysis}

Answers to the questionnaire were transferred to Excel (Microsoft Corp., Redmond, WA) and coded by the type of response. Descriptive and statistical analyses were conducted using JMP software version 4.0 and SAS 9.4 (SAS Institute, Cary, NC). For all analyses, a $P$-value of $<0.05$ was considered to be the threshold of significance.

Multiple Pearson chi-squared tests were first used to evaluate the association between the categorical variable of a WM sample being positive or negative for at least 1 drug residue and the various categorical variables of farm management practices and dairy characteristics generated through the on-farm questionnaire. These variables included antimicrobial drug used to treat cows for dry treatment, mastitis, reproductive disease, lameness and pneumonia, how antimicrobial treatments are recorded, range of number of cows milked into the WM bulk tank, and whether cows not treated with antimicrobials are milked into the WM bulk tank. Variables with a significant $P$-value were added to multiple logistic regression models to investigate associations between farm management practices and dairy characteristics as the independent variables and the presence of drug residues in WM as the dependent dichotomous variable. A Pearson chi-squared test was used to assess goodness of fit of the logistic regression models.

A 1-way ANOVA was used to evaluate associations between mean values of milk quality parameters and the presence of drug residues in WM. The independent dichotomous variables were the presence or not of at least 1 drug residue, or only cephalosporin drug residues, or only tetracycline drugs residues. The dependent variables of interest were the continuous variables from milk quality testing and included fat (\%), protein (\%), lactose (\%), SNF (\%), SCC (cells/mL), coliform count $(\mathrm{cfu} / \mathrm{mL})$, and SPC (cells/mL). Levene's test was used to test the equal variances assumption, and a Wilcoxon rank-sum test was used to compare group means in place of any linear model that violated this assumption.

Multiple Pearson chi-squared tests were used to evaluate the association between the categorical variable of a WM sample being positive or negative for 
at least 1 drug residue, 1 cephalosporin drug residue, and 1 oxytetracycline drug residue and the categorical variables for presence or not of culture-positive results for each microbe cultured from milk. Variables with a significant $P$-value were reported using relative risk for the risk of WM samples positive for a residue being culture positive when compared with the risk of the WM sample positive for this same drug residue being culture negative for the referred microbe.

\section{RESULTS}

\section{Farm Characteristics}

Farms were categorized based on the combined number of lactating and dry cows: 200 to 499 cows $(\mathrm{n}=2)$, 500 to 999 cows $(\mathrm{n}=4), 1,000$ to 2,999 cows $(\mathrm{n}=12)$, 3,000 to 5,000 cows $(\mathrm{n}=5)$, and $>5,000$ cows $(\mathrm{n}=2)$. The number of preweaned dairy calves present on the farm ranged between 40 and 64 calves $(\mathrm{n}=1), 65$ and 199 calves $(\mathrm{n}=5), 200$ and 499 calves $(\mathrm{n}=6), 500$ and 999 calves $(\mathrm{n}=4)$, and $>1,000$ calves $(\mathrm{n}=3)$. Of the 25 farms sampled, 5 raised heifer replacements off-site. The combined number of cows milked into the WM bulk tank was 1 to 9 cows ( $\mathrm{n}=5$ herds), 10 to 29 cows $(\mathrm{n}=10), 30$ to 59 cows $(\mathrm{n}=6), 60$ to 89 cows $(\mathrm{n}=3)$, and 90 to 119 cows $(n=1)$. The majority of the farms $(\mathrm{n}=18,72 \%)$ milked both treated and fresh cows into the WM bulk tank, whereas the remaining farms $(\mathrm{n}=$ $7,28 \%$ ) stored WM from treated cows and fresh cows separately.

\section{Questionnaire}

A total of 23 dairy farms (92\%) reported that all antibiotic treatments were recorded for each individual animal. Of the 23 farms that reported keeping a complete record of antibiotic treatments, $16(70 \%)$ recorded antimicrobial treatments using computerized dairy management software and $7(30 \%)$ maintained written records in a book. Distribution of farms using dairy management software versus a written record by number of lactating cows was, respectively, $0 \%$ versus $38 \%$ for 200 to 499 cows, $6 \%$ versus $38 \%$ for 500 to 999 cows, $56 \%$ versus $28 \%$ for 1,000 to 2,999 cows, $31 \%$ versus $0 \%$ for 3,000 to 5,000 cows, and $6 \%$ versus $14 \%$ for $>5,000$ cows.

Of all the study dairies, $12(48 \%)$ routinely used a teat sealant at dry-off. However, almost all farms used intramammary antimicrobial treatment at dry-off $(\mathrm{n}=$ $24,96 \%)$. In farms using intramammary antimicrobial treatment at dry-off, 21 farms $(87.5 \%)$ applied a blanket treatment program and $3(12.5 \%)$ used selective dry cow treatment. For the farms using selective dry cow treatment, $\mathrm{SCC}(\mathrm{n}=3)$ and days pregnant $(\mathrm{n}=1)$ were the factors considered when choosing which cows to treat. The most common antimicrobial used for intramammary dry-cow treatment was ceftiofur $(\mathrm{n}=12$, $48 \%$ ) followed by cephapirin $(\mathrm{n}=5,20 \%)$, penicillin ( $\mathrm{n}$ $=5,20 \%)$, and cloxacillin $(\mathrm{n}=2,8 \%)$.

The classes of antimicrobials used to treat lactating cows in the study farms were $\beta$-lactams, tetracyclines, sulfonamides, and lincosamides (Table 1). Intramammary ceftiofur was the most common drug used to treat mastitis during lactation $(68 \%, \mathrm{n}=17)$. Ceftiofur was also the most common drug used to treat reproductive diseases $(76 \%, \mathrm{n}=19)$, pneumonia $(68 \%, \mathrm{n}=17)$, and lameness $(40 \%, \mathrm{n}=10)$ that warranted systemic antimicrobial treatment. Reproductive disease included metritis, retained placenta, clinical endometritis, or other diseases related to reproduction for which the producer opted to treat an animal using antimicrobial drugs. No significant associations $(P>0.05)$ were identified between farm characteristics, management practices, and presence of drug residues in WM.

\section{Calf Feeding Management Practices}

Information regarding calf-rearing practices for those farms was not collected from the 5 farms that raised replacement heifers off-site. For the 20 farms that raised replacement heifers on-site, 19 (95\%) used WM as a feed for preweaned calves. The 1 exception sold WM to a nearby calf ranch, where the WM was fed to preweaned dairy calves. A total of 13 (65\%) farms fed milk replacer, either mixed with WM or administered alone for specific calf age groups. Of these farms that fed milk replacer, 7 (53.8\%) used nonmedicated milk replacer, $3(23.1 \%)$ added neomycin, and $3(23.1)$ added chlortetracycline. A total of 8 farms (40\%) fed saleable milk to preweaned dairy calves. Of the dairies raising preweaned calves, $15(75 \%)$ routinely pasteurized the milk before feeding. Methods of pasteurization included low temperature, long time $\left(62^{\circ} \mathrm{C}\right.$ for $30 \mathrm{~min}$, $\mathrm{n}=7)$; high temperature, short time $\left(71^{\circ} \mathrm{C}\right.$ for $15 \mathrm{~s}, \mathrm{n}$ $=7)$; and UV light $(\mathrm{n}=1)$. The mean age at weaning was 9 wk.

\section{Quantification of Drug Residues Using LC-MS/MS}

Of 25 milk samples submitted for LC-MS/MS, 15 $(60 \%)$ had detectable concentrations of 1 or more of the 27 drug residues screened (Table 2). The classes of antimicrobials found in the WM samples were $\beta$-lactams $(\mathrm{n}=11)$, tetracyclines $(\mathrm{n}=4)$, and sulfonamides ( $\mathrm{n}$ $=1$ ). Only 1 sample of the 15 positive samples con- 
Table 1. Responses to an on-farm questionnaire regarding antimicrobial drug use preferences for dry cow treatment, mastitis, reproductive diseases, lameness, and pneumonia on a convenience sample of California dairy farms $(\mathrm{n}=25)^{1}$

\begin{tabular}{|c|c|c|c|c|c|}
\hline Antimicrobial agent & $\begin{array}{l}\text { Dry cow } \\
\text { treatment }\end{array}$ & Mastitis & $\begin{array}{c}\text { Reproductive } \\
\text { diseases }\end{array}$ & Lameness $^{2}$ & Pneumonia \\
\hline$\overline{\text { Amoxicillin }}$ & & $1^{3}$ & $7^{3}$ & $2^{3}$ & 4 \\
\hline Cephapirin & 6 & 10 & & & \\
\hline Cloxacillin & 3 & 1 & & & \\
\hline Oxytetracycline & & & 5 & 2 & 5 \\
\hline Penicillin G & 6 & 1 & $9^{3}$ & $8^{3}$ & 3 \\
\hline Tetracycline & & & & 19 & \\
\hline Penicillin + sulfadimethoxine & & & & 1 & \\
\hline Oxytetracycline + sulfadimethoxine & & & & & 1 \\
\hline Florfenicol & & & & & 2 \\
\hline No treatment ${ }^{4}$ & & & & & 4 \\
\hline
\end{tabular}

${ }^{1}$ Some farms reported 2 antimicrobials for treating cows.

${ }^{2}$ This included a combination of both parenteral and topical antimicrobials used to treat cows diagnosed with lameness disease.

${ }^{3}$ Antimicrobial drugs that do not have any commercial drug label that includes the referred disease as an indication for use.

${ }^{4}$ Farms that reported not having a drug preference for treating that specific disease.

tained both $\beta$-lactams and tetracyclines, whereas the remaining 14 were positive for only 1 class of antibiotic. Ceftiofur was the most frequently detected drug residue [present in $7(28 \%)$ of the WM samples], followed by oxytetracycline [present in $4(16 \%)$ of the samples] and cephapirin [found in 3 (12\%) of the samples; Table 3]. The FDA tolerance and safe limit for drug residues in raw milk is displayed in Table 3. One sample had detectable concentrations of penicillin G, 1 had detectable concentrations of sulfadimethoxine, and 1 had detectable concentrations of tetracycline. No samples had detectable concentrations of 5-hydroxyflunixin. Ten (40\%) out of 25 milk samples submitted for LC-MS/ MS had no detectable concentrations of any of the 27 drug residues screened.

\section{Milk Quality Parameters}

The mean fat (\%), protein (\%), lactose (\%), SNF (\%), SCC, coliform count, and SPC for WM samples are displayed in Table 4. Significant differences in coliform count $(P=0.009)$ and $\operatorname{SPC}(P=0.01)$ were found only between WM samples positive for tetracycline class residues compared with samples negative for tetracycline residues (Table 5).

\section{Milk Microbiology}

The most common genera of bacteria isolated from WM samples was Streptococcus spp. $(\mathrm{n}=21,84 \%)$ followed by Staphylococcus spp. $(\mathrm{n}=20,80 \%)$ and E. coli ( $\mathrm{n}=10,40 \%$; Table 6$)$. Other major mastitis pathogens isolated were Staphylococcus aureus ( $\mathrm{n}=5,20 \%)$, Klebsiella spp. ( $\mathrm{n}=4,16 \%)$, and Mycoplasma spp. (n
$=2,8 \%)$. Culture-positive $S$. aureus WM samples were significantly associated with samples being positive for at least 1 drug residue in WM $(P=0.04)$ when compared with WM samples negative for any drug residues in WM. The relative risk for a WM sample positive for at least 1 drug residue being culture positive for $S$. aureus was 2.0 (95\% CI: 1.2-3.1) compared with being culture negative for $S$. aureus.

Similarly, the probability of a WM sample being positive for $S$. aureus was significantly higher $(P=0.04)$ if the sample was positive for cephalosporin drug residues compared with WM samples negative for cephalosporin residues. In addition, an increased probability for culturing Mycoplasma spp. was significantly associated $(P=0.04)$ with WM samples containing drug residues for cephalosporin residues when compared with WM samples negative for cephalosporin residues. The rela-

Table 2. Distribution of antimicrobial drug residues in raw waste milk from a convenience sample of California dairies $(n=25)^{1}$

\begin{tabular}{lc}
\hline Description $^{2}$ & $\begin{array}{c}\text { Frequency } \\
(\%)\end{array}$ \\
\hline $\begin{array}{l}\text { Result of commercial LC-MS/MS test }(\mathrm{n}=25) \\
\text { Positive }\end{array}$ & $15(60)$ \\
Negative & $10(40)$ \\
Distribution by drug class (by LC-MS/MS; $\mathrm{n}=25)$ & $10(40)$ \\
Negative & $10(40)$ \\
Only $\beta$-lactams & $1(4)$ \\
Only sulfamethazine & $3(12)$ \\
Only tetracyclines & $1(4)$ \\
\hline -Lactams and tetracycline & \\
\hline
\end{tabular}

${ }^{1}$ Residues were detected by liquid chromatography-tandem MS (LCMS/MS).

${ }^{2}$ Positive $=$ milk samples tested positive for at least 1 of the drugs tested; negative $=$ milk samples tested negative for all of the drugs tested. 
Table 3. Antimicrobial drug residues in waste milk from a convenience sample of California dairies $(\mathrm{n}=25)$ as measured by liquid chromatography-tandem MS (LC-MS/MS)

\begin{tabular}{lccccc}
\hline Drug residue & $\%(\text { no. })^{1}$ & $\begin{array}{c}\text { Mean } \\
(\mathrm{ng} / \mathrm{mL})\end{array}$ & $\mathrm{SE}$ & $\begin{array}{c}\mathrm{LOQ}^{2} \\
(\mathrm{ng} / \mathrm{mL})\end{array}$ & $\begin{array}{c}\text { Tolerance }^{3} \\
(\mathrm{ng} / \mathrm{mL})\end{array}$ \\
\hline $\begin{array}{l}\beta \text {-Lactams } \\
\text { Ceftiofur }\end{array}$ & $44(11)$ & & & & \\
Cephapirin & $28(7)$ & 251.14 & 177.0 & $<10$ & 100 \\
$\begin{array}{l}\text { Penicillin G } \\
\text { Sulfonamides }\end{array}$ & $12(3)$ & 39 & 24.5 & $<10$ & 20 \\
$\quad$ Sulfadimethoxine & $4(1)$ & 10 & - & $<5$ & 0 \\
Tetracyclines & $4(1)$ & 7 & - & $<2$ & 300 \\
$\quad$ Tetracycline & $16(4)$ & & & $<10$ & 300 \\
Oxytetracycline & $4(1)$ & 590 & - & $<10$ & 0
\end{tabular}

${ }^{1}$ Number of milk samples with antimicrobial drug residues at concentrations above the limit of quantification for LC-MS/MS for each individual drug or drug class described.

${ }^{2}$ Limit of quantification of LC-MS/MS for each drug detected in the milk samples.

${ }^{3}$ Food and Drug Administration tolerance or safe limit for drug residues in raw milk.

tive risk for a WM sample positive for cephalosporin drug residues being culture positive for $S$. aureus was 2.6 (95\% CI: $1.2-5.9)$ and for Mycoplasma was 2.8 (95\% CI: 1.6-5.0) when compared with being culture negative for these bacteria.

\section{Antimicrobial Resistance in E. coli Isolates}

Escherichia coli was isolated from 10 (40\%) WM samples. Of the $10 \mathrm{E}$. coli isolates that were tested against selected antimicrobials, $70 \%$ were susceptible to $\beta$-lactams and tetracyclines and $80 \%$ were susceptible to sulfonamides (Table 7). Prevalence of multiple drug resistance was $20 \%$. Among the $\beta$-lactams tested, 3 isolates were resistant to ampicillin and 2 were resistant to cephalothin. One isolate was resistant to ceftiofur, and 2 isolates showed intermediate susceptibility. Only $40 \%$ of the isolates were sensitive to all antimicrobials tested.

\section{DISCUSSION}

\section{Quantification of Drug Residues}

Ceftiofur was the most common drug detected in WM and is the only third-generation cephalosporin licensed to treat food-producing animals in the United States. Parenteral administration of commercially available ceftiofur at the approved label dosage does not result in milk residues at concentrations above the tolerance levels established by the FDA. Ceftiofur residues are likely from the use of an intramammary treatment of a cow for mastitis. According to the label, intramammary ceftiofur has a withdrawal period of $72 \mathrm{~h}$ after the last treatment before milk from a treated cow may be sold for human consumption.

In a study by Pereira et al. (2014a), feeding preweaned calves milk containing a very low concentration of antimicrobials for $6 \mathrm{wk}$ resulted in selection of multidrug-resistant $E$. coli in their feces when compared with the control group fed milk without drug residues. In the Pereira et al. (2014a) study, milk fed to calves in the treatment group was spiked with ceftiofur, penicillin, ampicillin, and oxytetracycline at concentrations of 100, 5, 10, and $300 \mathrm{ng} / \mathrm{mL}$, respectively. Except for ampicillin, all these drugs were detected in WM of dairy farms in California at similar concentrations. Furthermore, the Pereira et al. (2014a) study supports the hypothesis that, on average, if calves in California are fed WM, we can expect to observe a higher prevalence of antimicrobial resistant (AMR) enteric bacteria in

Table 4. Milk quality parameters for waste milk samples collected from a convenience sample of California dairies $(\mathrm{n}=25)$

\begin{tabular}{lccc}
\hline Variable & Mean & \multicolumn{1}{c}{ SD } & \multicolumn{1}{c}{$95 \%$ CI } \\
\hline Milk fat (\%) & 4.24 & 1.41 & $3.66,4.82$ \\
Milk protein $(\%)$ & 3.74 & 0.43 & $3.56,3.92$ \\
Lactose (\%) & 4.4 & 0.22 & $4.31,4.49$ \\
SNF $(\%)$ & 8.77 & 0.45 & $8.59,8.96$ \\
SCC $\left(\times 10^{3} \mathrm{cells} / \mathrm{mL}\right)$ & $2,133.60$ & $1,260.14$ & $1,613.44,2,653.76$ \\
Coliforms $(\mathrm{cfu} / \mathrm{mL})$ & 702.4 & 691.12 & $417.12,987.68$ \\
SPC $\left(\times 10^{3} \mathrm{cfu} / \mathrm{mL}\right)$ & 116.27 & 101.19 & $74.50,158.04$ \\
\hline
\end{tabular}


Table 5. Comparison of milk quality parameters for waste milk samples from a convenience sample of California dairies $(\mathrm{n}=25)$ stratified by test status for at least 1 antimicrobial drug residue, ceftiofur drug residues, and oxytetracycline drug residues

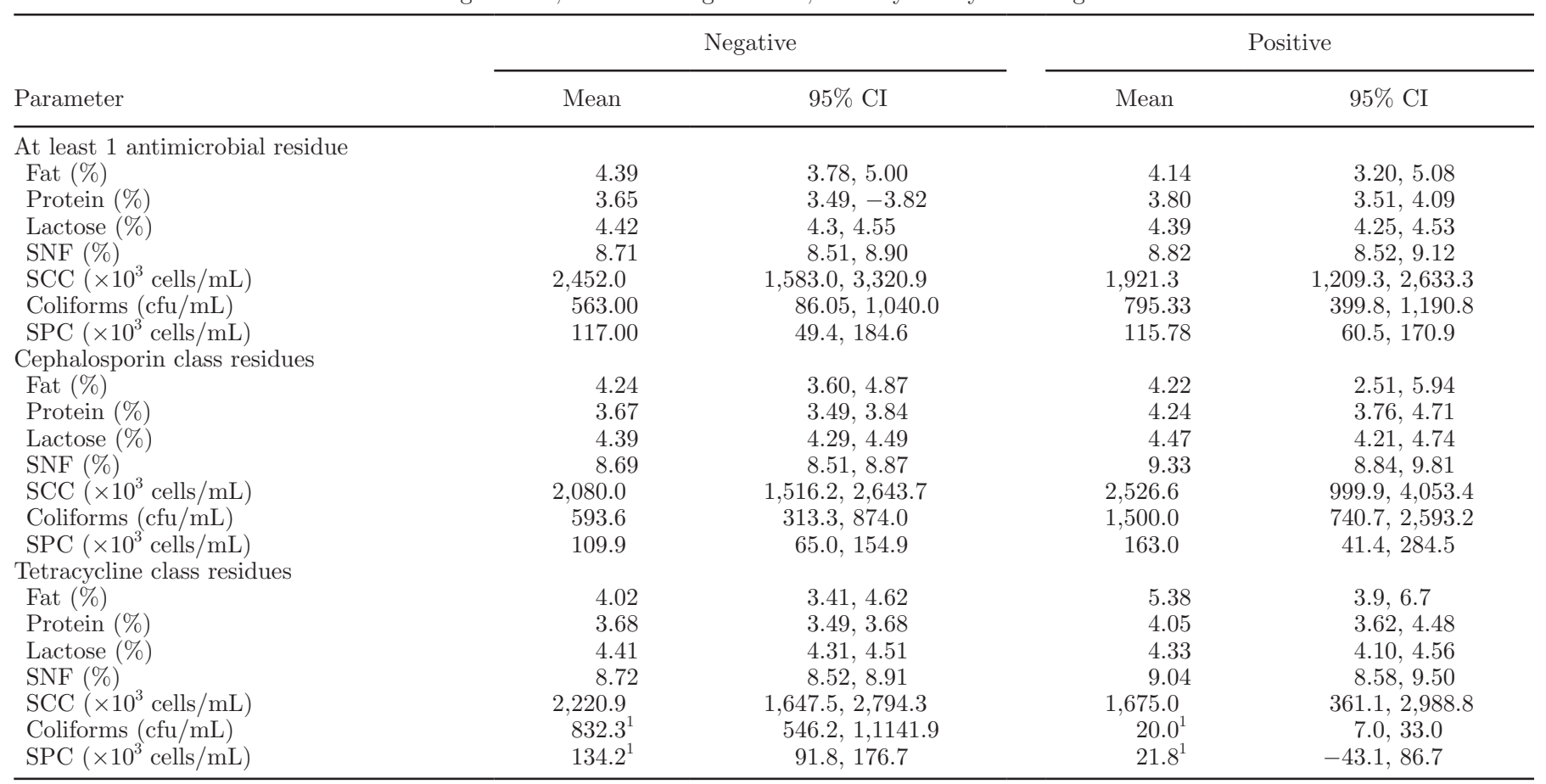

${ }^{1}$ Mean value and 95\% confidence interval for the 1-way ANOVA used to evaluate samples positive or negative for presence of a specific drug residue.

their feces compared with calves fed milk not containing antimicrobial residues.

Compared with 2 other studies that evaluated drug residues in milk, the first conducted in the Central Valley of California (Selim and Cullor, 1997) and the second conducted in New York State (Pereira et al., 2014b), our results were within the range expected for observing drug residues in WM. From these 2 studies, the first showed an overall prevalence of drug residues in WM of $63 \%$, whereas the second showed that $82 \%$ of
WM samples had at least 1 drug residue at detectable concentrations. In both studies, the overall prevalence of antimicrobial drug residues in WM was calculated using commercial SNAP tests. In the New York study, all the positive samples on SNAP tests were submitted for drug identification and quantification using LCMS/MS. In our study, all the WM samples collected were tested with LC-MS/MS, and we found an overall prevalence of $60 \%$ of WM samples positive for at least 1 of the antimicrobials tested. The lower prevalence

Table 6. Culture results for waste milk samples collected from a convenience sample of California dairies (n $=25)$

\begin{tabular}{lccr}
\hline Bacteria & $\begin{array}{c}\text { No. (\%) culture } \\
\text { positive }\end{array}$ & $\begin{array}{c}\text { Mean cfu } \\
\left(\times 10^{3} / \mathrm{mL}\right)\end{array}$ & SD $^{1}$ \\
\hline Streptococcus spp. & $21(84)$ & 51.01 & 89.96 \\
Staphylococcus spp. & $20(80)$ & 8.63 & 22.09 \\
Escherichia coli & $10(40)$ & 5.87 & 7.83 \\
Nonfermentative gram negative & $8(32)$ & 9.63 & 8.73 \\
Staphylococcus aureus & $5(20)$ & 121.40 & 267.55 \\
Bacillus spp. & $5(20)$ & 0.86 & 1.26 \\
Klebsiella spp. & $4(16)$ & 20.75 & 147.54 \\
Trueperella pyogenes & $3(12)$ & - & - \\
Mycoplasma spp. & 26.01 \\
Enterobacter spp. & $2(8)$ & 10 & - \\
Prototheca spp. & $1(4)$ & 3 & - \\
Yeasts & $1(4)$ & 10 & - \\
\hline
\end{tabular}

${ }^{1}$ Standard deviation of the mean for milk colony-forming units per milliliter.

${ }^{2}$ No quantification of Mycoplasma culture-positive samples was conducted. 
Table 7. Distribution of antimicrobial susceptibility testing of Escherichia coli isolated from waste milk samples $(\mathrm{n}=10)$ from a convenience sample of California dairies $(\mathrm{n}=25)$

\begin{tabular}{lccc}
\hline Antimicrobial & $\begin{array}{c}\%(\text { no. } \\
\text { susceptible }\end{array}$ & $\begin{array}{c}\%(\text { no. } \\
\text { intermediate }\end{array}$ & $\begin{array}{c}\% \text { (no.) } \\
\text { resistant }\end{array}$ \\
\hline$\beta$-Lactams & $70(7)$ & $0(0)$ & $30(3)$ \\
Ampicillin & $80(8)$ & $0(0)$ & $20(2)$ \\
Cephalothin & $70(7)$ & $20(2)$ & $10(1)$ \\
Ceftiofur & $70(7)$ & $0(0)$ & $30(3)$ \\
Tetracycline & $80(8)$ & $0(0)$ & $20(2)$ \\
$\quad \begin{array}{l}\text { Tetracycline } \\
\text { Sulfonamides }\end{array}$ & & & \\
\hline
\end{tabular}

of positive samples may have been due to the higher limit of detection of the LC-MS/MS compared with the commercial SNAP tests. In both previous studies, the most common classes of antimicrobial residues were $\beta$-lactams followed by tetracyclines. Similarly, in the present study, the $\beta$-lactam class was the most common antimicrobial class and the tetracycline class was the second most common. Common use of these drugs on dairy farms may be the reason for the higher prevalence of residues of these drugs.

A survey conducted on 113 dairy farms in Pennsylvania (Sawant et al., 2005) found that $\beta$-lactams and tetracyclines were the most widely used antibiotics. Despite 14 antimicrobials belonging to 8 different classes having approved use for adult lactating dairy cows in the United States, in the present study we found only 6 antimicrobial drug residues in WM belonging to 3 different classes.

\section{On-Farm Antimicrobial Use}

Ceftiofur was the most commonly reported antimicrobial used for all the disease categories evaluated (intramammary dry-off treatment, mastitis during lactation, reproductive disease, pneumonia, and nontopical lameness treatment). This result confirms findings from other surveys on antimicrobial use on dairy farms (Zwald et al., 2004). Although ceftiofur is used only in veterinary medicine, all third-generation cephalosporins have been classified as critically important antimicrobials in human medicine by the World Health Organization (WHO, 2007), and the use of ceftiofur in food animals represents a major public health concern because of the possible development of resistance to other extended-spectrum cephalosporins, such as ceftriaxone and cephamycins, used in human medicine (Dunne et al., 2000). Due to the importance of ceftiofur as a veterinary drug as well as a drug class of importance to human medicine, the extralabel use of ceftiofur in the United States is limited, and according to the FDA it can only be used extralabel to treat or control an extralabel disease indication as long as this use adheres to a labeled dosage regimen (i.e., dose, route, frequency, and duration of administration) approved for that particular species and production class (FDA, 2018b). Furthermore, unnecessary extralabel use of antimicrobials considered of critical importance such as third-generation cephalosporin drugs, especially when an antimicrobial belonging to a class of lower importance to veterinary and human medicine could be used, can result in unnecessary selection of antimicrobial resistance to important drug classes (FDA, 2003).

Tetracycline was the treatment of choice for topical antimicrobial treatment of lameness in $60 \%$ of the farms. The only nonapproved antimicrobial used on the study dairies was florfenicol, which was routinely used by 2 dairy producers to treat pneumonia in adult cows. Florfenicol is not approved for use in dairy cattle $\geq 20$ mo of age (Smith et al., 2005); however, it may be used in an extralabel fashion in food-producing species (Payne et al., 1999; Davis et al., 2009) by a licensed veterinarian or a layperson under the supervision of a licensed veterinarian within the scope of a valid veterinary client-patient relationship and with appropriate prescription and labeling.

Keeping adequate records of antimicrobial drug treatment is a very important routine practice for farms to ensure a low risk of cows with milk containing antimicrobial drug residues above tolerance or safe levels being milked into the saleable milk bulk tank. Our study questionnaire reported that 2 out of the 25 farms did not have complete records on cow antimicrobial treatment. The FDA views the failure of a dairy farm to keep adequate drug treatment records as an inadequate control measure that creates unsanitary conditions, and therefore adulterated food, under the US Federal Food, Drug, and Cosmetic Act (FDA, 2018a). Most of the farms in the study kept records using a farm management computer program (70\%). This is in agreement with the NAHMS report on dairy cattle management, where $60.8 \%$ of medium (100-499 cows) and $98 \%$ of large ( $\geq 500$ cows) farms reportedly used a computer system for record keeping, which included use of antimicrobials (USDA, 2016). Keeping adequate drug treatment records using a computerized system can be an effective method to monitor individual animal treatment and generate reports on overall usage of antimicrobials on the farm. A guideline for adequate record keeping can be found on the FDA website and includes keeping a record of the name of the drug used, individual identity of the animal treated, date of each administration of the drug to the animal, dosage used, and route of administration (FDA, 2018a). This information can be a useful tool to monitor disease 
incidence over time and improve decision making on investment in preventive measures that could reduce disease risk and antimicrobial drug use on the farm. To the authors' knowledge, the FDA does not currently consider computer records as an inadequate method to record individual animal antimicrobial treatments (FDA, 2018a); the exception is that the FDA requires a farm to have written records after a drug residue violation (FDA, 2011b). Furthermore, digital records can be printed when needed to generate hard copies of that information.

Although our study questionnaire collected relevant information that could result in drug residues being detected in WM, questions were mostly limited to practices directly linked to selection of drugs used to treat the most common diseases observed in dairy cattle. Therefore, there is a need for future research that collects more extensive information about herd management to allow identification of practices that could be addressed in order to reduce unnecessary treatment of cattle with antimicrobial drugs and therefore decrease antimicrobial residues in milk.

\section{Milk Quality Parameters}

Most of the study farms housed and milked both treated and postpartum cows together. Therefore, the WM samples were a blend of colostrum, transition milk, and other nonsaleable milk. Consequently, the mean fat, protein, and lactose contents were intermediate between the standard composition of the different types of milk reported in the literature (Godden, 2008). In our study, we found high variability in the SCC in WM samples, likely reflecting different management practices related to hospital and fresh cows between different farms where WM samples were collected. High SCC values and high variability in the composition of WM have been previously reported in the literature (Moore et al., 2009) and suggested to be related to milk from mastitic cows with high SCC increasing the average SCC in the WM bulk tank. A study evaluating SPC and coliform counts in more than 150 colostrum samples from 40 dairy farms observed that SPC and coliform counts exceeded 1,000,000 and 10,000 cfu/mL, respectively, in $85 \%$ of the samples tested (McGuirk and Collins, 2004). We also observed high SPC and coliform counts in our study, with mean values above 111,000 and $700 \mathrm{cfu} / \mathrm{mL}$, respectively.

The only drug class for which residues in WM had significant effects on the SPC and coliform counts was tetracycline, which resulted in significantly lower concentrations for both bacteriological measures when compared with WM samples not containing tetracycline residues. A hypothesis for this finding is that the concentration of tetracycline drugs in WM samples was significantly reducing the replication of culturable bacteria in WM samples. Although the MIC of tetracycline for $E$. coli, a common gram-negative environmental bacteria, is $1,000 \mathrm{ng} / \mathrm{mL}$, exposure of gram-negative bacteria to tetracycline concentrations below the MIC has been shown to affect growth rates as well as the dynamics of selection of antimicrobial-resistant bacteria (Gullberg et al., 2011). A potential explanation for why other drug residues (e.g., cephalosporins) in WM did not similarly affect SPC and coliform counts could be because they were present at lower mean concentrations compared with the tetracycline drugs and because cephalosporin MIC for common gram-negative bacteria such as $E$. coli is 2 times greater $(2,000 \mathrm{ng} / \mathrm{mL})$ than that of tetracycline.

\section{Isolation and Identification of Bacteria}

Selim and Cullor (1997) found a significantly higher number of bacteria in WM compared with other types of milk. In our study, we found high numbers of bacteria, which may be due to the fact that most farms stored milk in nonrefrigerated tanks before feeding it to calves. Given that WM can originate from cows treated with antimicrobials, the risk of AMR bacteria in WM could be from direct selection of AMR bacteria from the cow as a consequence of drug treatment as well as further selection of AMR bacteria in bulk tank milk due to the selective pressure caused by drug residues present in that environment (Sandegren, 2014; Awosile and Smith, 2017). Although pasteurization may reduce the bacteria load of WM, limited information is available on approaches to degrade drug residues in WM to reduce selection of AMR bacteria postpasteurization due to the presence of drug residues.

Waste milk samples cultured positive for $S$. aureus were significantly more commonly identified in samples positive for cephalosporin drugs compared with negative samples. Similarly, Mycoplasma spp.--positive samples had a significantly higher probability of being cultured in samples positive for cephalosporin drugs compared with negative samples. Both S. aureus and Mycoplasma bovis are contagious bacteria that cause mastitis and result in a significant cost to the dairy industry because of the low cure rate (S. aureus) and the lack of effective antimicrobial drugs (Mycoplasma spp.) to treat these pathogens, resulting in many cases of culling of animals from the herd (Barkema et al., 2006; Royster and Wagner, 2015). The higher concentration of cephalosporin drugs in milk samples that cultured positive for these 2 bacteria could be due to the spread of mastitis caused by these contagious bacteria, resulting in greater numbers of cows being treated for clinical mastitis, as well 
as an increase in chronic cases of mastitis, resulting in larger numbers of cows with cephalosporin drug residues in milk being withheld from human consumption. Use of intramammary antimicrobials to treat mastitis without diagnostic information on the pathogen causing mastitis is a good example of how erroneous or lack of diagnostic information can result in greater cost to the dairy. This increased cost could be from antimicrobial drug costs, milk discard, disease transmission risk, and the potential for transmission of antimicrobial drug-resistant pathogens (e.g., Mycoplasma spp.) via WM (Pereira et al., 2014a; Cha et al., 2016). Furthermore, presence of Mycoplasma spp. in WM used as a feed source may be detrimental to calf health and may result in higher occurrence of Mycoplasma pneumonia and otitis media in calves (Walz et al., 1997).

\section{Antimicrobial Resistance in E. coli Isolates}

In the present study, we found both detectable concentrations of drug residues in the milk and presence of resistant bacteria. Because we focused our analysis on $E$. coli isolates, these resistant bacteria more likely come from environmental or fecal contamination of the WM during harvest and storage. Even in those cases, $E$. coli isolates that originate from the environment may be exposed to selective pressure in the WM due to antimicrobial residues (Ricci et al., 2017). Although no significant association between the presence of drug residues in WM samples and AMR in the E. coli isolates was found, no quantitative methods for evaluating the prevalence of AMR were used in the study, and we could not separate AMR isolates originating from cows being treated with antimicrobials versus selection of AMR bacteria caused by drug residues in the WM bulk tank. The low number of samples that cultured positive for $E$. coli warrants further studies that focus on evaluating risk factors of AMR bacteria selection in WM samples. The presence of multidrug-resistant $E$. coli in WM urges the need for on-farm practices, such as pasteurization, that reduce the risk of exposure to calves and dissemination of resistant bacteria into the environment (Awosile and Smith, 2017).

One of the limitations of the current study is the cross-sectional study design, where data collection occurred only at a single point, limiting causal inference between exposures of interest (on-farm management practices) and outcomes (drug residues in WM). Longitudinal studies are required to study the causal associations between management practices and antimicrobial resistance and provide further information on the effects of feeding preweaned calves WM. A controlled trial was previously conducted in which calves were fed raw saleable milk from birth to weaning, with half of calves receiving milk spiked with 4 antibiotics, including ceftiofur, at the tolerance levels established by the FDA and the other half fed the same raw saleable milk but without the addition of any antimicrobials. This controlled trial showed a significant increase in the proportion of fecal shedding of $E$. coli resistant to ceftiofur as well as multidrug-resistant bacteria (Pereira et al., 2014a). One limitation of that study was that it did not take into account potential on-farm effects; however, it provides scientific evidence of how low drug residue concentrations can select for antimicrobial-resistant bacteria.

The novel information generated by this study will help guide future studies focused on addressing the issue of feeding milk containing drug residues to calves using data that improved the validity of conclusions made specifically for farms in Central California. Concerns worldwide with this practice can be observed in the recent report by the European Food Safety Authority evaluating the risk for development of antimicrobial resistance due to feeding calves milk that contains residues of antibiotics (EFSA, 2017). With increased concerns about unnecessary exposure of animals to antimicrobial drugs, especially those present in feed, and the current ambiguous legality of feeding calves milk containing drug residues in the United States, scientific information is of critical importance to support future decisions and interventions that may affect the practice of feeding WM to dairy calves.

\section{CONCLUSIONS}

Waste milk fed to calves on dairies in California's Central Valley contains residues of antimicrobial drugs that are important to veterinary and human medicine. The presence of drug residues in WM at concentrations that increase the selection of resistant bacteria indicates the need for additional studies targeting on-farm milk treatments to degrade drug residues before feeding to calves. This also highlights the importance of antimicrobial stewardship and proper preventive disease management practices that reduce disease risk and the need for antimicrobial drug use in lactating dairy cows. The presence of multidrug-resistant E. coli in WM urges the need for on-farm practices, such as pasteurization, that reduce calf exposure to resistant bacteria.

\section{ACKNOWLEDGMENTS}

Funding was provided by USDA (Washington, DC) project number CA-V-PHR-4708-AH408 and University of California, Davis Division of Agriculture and 
Natural Resources grant number 1753. Any opinions, findings, conclusions, or recommendations expressed in this publication are those of the authors and do not necessarily reflect the view of the USDA. The authors acknowledge the participating dairy owners, herd managers, and veterinary practitioners. The authors also thank Kathy Glenn and Karen Tonooka from the Milk Quality Laboratory at the Veterinary Medicine Teaching and Research Center (Tulare, CA) for culture testing of milk samples.

\section{REFERENCES}

Awosile, B. B., and B. A. Smith. 2017. Risk assessment modelling of fecal shedding caused by extended-spectrum cephalosporin-resistant Escherichia coli transmitted through waste milk fed to dairy pre-weaned calves. J. Dairy Sci. 100:9667-9673.

Barkema, H. W., Y. H. Schukken, and R. N. Zadoks. 2006. Invited review: The role of cow, pathogen, and treatment regimen in the therapeutic success of bovine Staphylococcus aureus mastitis. J. Dairy Sci. 89:1877-1895.

CDFA (California Department of Food and Agriculture). 2017. California Dairy Statistics Annual: 2016 Data. CDFA, Sacramento, CA.

Cha, E., R. L. Smith, A. R. Kristensen, J. A. Hertl, Y. H. Schukken, L. W. Tauer, F. L. Welcome, and Y. T. Grohn. 2016. The value of pathogen information in treating clinical mastitis. J. Dairy Res. 83:456-463.

CLSI (Clinical and Laboratory Standards Institute). 2013. Performance Standards for Antimicrobial Disk and Dilution Susceptibility Tests for Bacteria Isolated From Animals. Approved Standard. 4th ed. CLSI Document No. VET01-A4. CLSI, Wayne, PA.

CLSI (Clinical and Laboratory Standards Institute). 2015. Performance Standards for Antimicrobial Susceptibility Testing. 25th Informational Supplement. CLSI Document No. M100-S25. CLSI, Wayne, PA.

Davis, J. L., G. W. Smith, R. E. Baynes, L. A. Tell, A. I. Webb, and J. E. Riviere. 2009. Update on drugs prohibited from extralabel use in food animals. J. Am. Vet. Med. Assoc. 235:528-534.

Dunne, E. F., P. D. Fey, P. Kludt, R. Reporter, F. Mostashari, P. Shillam, J. Wicklund, C. Miller, B. Holland, K. Stamey, T. J. Barrett, J. K. Rasheed, F. C. Tenover, E. M. Ribot, and F. J. Angulo. 2000. Emergence of domestically acquired ceftriaxone-resistant Salmonella infections associated with AmpC beta-lactamase. JAMA 284:3151-3156.

EFSA Panel on Biological Hazards (BIOHAZ), A. Ricci, A. Allende, D. Bolton, M. Chemaly, R. Davies, P. S. F. Escámez, R. Girones, K. Koutsoumanis, R. Lindqvist, B. Nørrung, L. Robertson, G. Ru, M. Sanaa, M. Simmons, P. Skandamis, E. Snary, N. Speybroeck, B. Ter Kuile, J. Threlfall, H. Wahlström, B. Bengtsson, D. Bouchard, L. Randall, B.-A. Tenhagen, E. Verdon, J. Wallace, R. Brozzi, B. Guerra, E. Liebana, P. Stella, and L. Herman. 2017. Risk for the development of antimicrobial resistance (AMR) due to feeding of calves with milk containing residues of antibiotics. EFSA J. 15:4665.

FDA (US Food and Drug Administration). 2003. Guidance for Industry no. 152: Evaluating the Safety of Antimicrobial New Animal Drugs with Regard to Their Microbiological Effects on Bacteria of Human Health Concern. FDA, Washington, DC.

FDA (US Food and Drug Administration). 2011a. Optimization and Validation of Multi-class, Multi-residue LC-MS/MS Screening and Confirmation Method for Drug Residues in Milk. FDA, Washington, DC.

FDA (US Food and Drug Administration). 2011b. A Win for FDA's Food Safety Mission. FDA, Washington, DC.
FDA (US Food and Drug Administration). 2018a. Adequate Drug Treatment Records Help Ensure Food Safety. FDA. Washington, DC

FDA (US Food and Drug Administration). 2018b. Extralabel Use and Antimicrobials. FDA, Washington, DC.

Godden, S. 2008. Colostrum management for dairy calves. Vet. Clin. North Am. Food Anim. Pract. 24:19-39.

Godden, S. M., J. P. Fetrow, J. M. Feirtag, L. R. Green, and S. J. Wells. 2005. Economic analysis of feeding pasteurized nonsaleable milk versus conventional milk replacer to dairy calves. J. Am. Vet. Med. Assoc. 226:1547-1554.

Gullberg, E., S. Cao, O. G. Berg, C. Ilback, L. Sandegren, D. Hughes, and D. I. Andersson. 2011. Selection of resistant bacteria at very low antibiotic concentrations. PLoS Pathog. 7:e1002158.

Maron, D. F., T. J. Smith, and K. E. Nachman. 2013. Restrictions on antimicrobial use in food animal production: An international regulatory and economic survey. Global. Health 9:48.

Maynou, G., A. Bach, and M. Terre. 2017a. Feeding of waste milk to Holstein calves affects antimicrobial resistance of Escherichia coli and Pasteurella multocida isolated from fecal and nasal swabs. J. Dairy Sci. 100:2682-2694.

Maynou, G., L. Migura-Garcia, H. Chester-Jones, D. Ziegler, A. Bach, and M. Terre. 2017b. Effects of feeding pasteurized waste milk to dairy calves on phenotypes and genotypes of antimicrobial resistance in fecal Escherichia coli isolates before and after weaning. J. Dairy Sci. 100:7967-7979.

McGuirk, S. M., and M. Collins. 2004. Managing the production, storage, and delivery of colostrum. Vet. Clin. North Am. Food Anim. Pract. 20:593-603.

Monson, J., K. Dapper, C. Chigbu, and L. Tang. 2015. California Dairy Statistics Annual D. M. B. Division of Marketing Services. 20 pages. Dairy Marketing Branch, Sacramento, CA. Accessed Jun. 26, 2018. www.cdfa.ca.gov/dairy/pdf/Annual/2015/2015 _Statistics_Annual.pdf.

Moore, D. A., J. Taylor, M. L. Hartman, and W. M. Sischo. 2009. Quality assessments of waste milk at a calf ranch. J. Dairy Sci. 92:3503-3509.

Oliver, S. P., S. E. Murinda, and B. M. Jayarao. 2011. Impact of antibiotic use in adult dairy cows on antimicrobial resistance of veterinary and human pathogens: A comprehensive review. Foodborne Pathog. Dis. 8:337-355.

Payne, M. A., R. E. Baynes, S. F. Sundlof, A. Craigmill, A. I. Webb, and J. E. Riviere. 1999. Drugs prohibited from extralabel use in food animals. J. Am. Vet. Med. Assoc. 215:28-32.

Pereira, R. V., S. Lima, J. D. Siler, C. Foditsch, L. D. Warnick, and R. C. Bicalho. 2016. Ingestion of milk containing very low concentration of antimicrobials: Longitudinal effect on fecal microbiota composition in preweaned calves. PLoS One 11:e0147525.

Pereira, R. V., J. D. Siler, R. C. Bicalho, and L. D. Warnick. 2014a. In vivo selection of resistant $E$. coli after ingestion of milk with added drug residues. PLoS One 9:e115223.

Pereira, R. V., J. D. Siler, R. C. Bicalho, and L. D. Warnick. 2014b. Multiresidue screening of milk withheld for sale at dairy farms in central New York State. J. Dairy Sci. 97:1513-1519.

Pereira, R. V. V., L. M. Carroll, S. Lima, C. Foditsch, J. D. Siler, R. C. Bicalho, and L. D. Warnick. 2018. Impacts of feeding preweaned calves milk containing drug residues on the functional profile of the fecal microbiota. Sci. Rep. 8:554.

Ricci, A., A. Allende, D. Bolton, M. Chemaly, R. Davies, P. S. Fernández Escámez, R. Girones, K. Koutsoumanis, R. Lindqvist, B. Nørrung, L. Robertson, G. Ru, M. Sanaa, M. Simmons, P. Skandamis, E. Snary, N. Speybroeck, B. T. Kuile, J. Threlfall, H. Wahlström, B. Bengtsson, D. Bouchard, L. Randall, B. A. Tenhagen, E. Verdon, J. Wallace, R. Brozzi, B. Guerra, E. Liebana, P. Stella, and L. Herman. 2017. Scientific opinion on the risk for the development of Antimicrobial Resistance (AMR) due to feeding of calves with milk containing residues of antibiotics. EFSA J. 15:4665.

Royster, E., and S. Wagner. 2015. Treatment of mastitis in cattle. Vet. Clin. North Am. Food Anim. Pract. 31:17-46. 
Sandegren, L. 2014. Selection of antibiotic resistance at very low antibiotic concentrations. Ups. J. Med. Sci. 119:103-107.

Sawant, A. A., L. M. Sordillo, and B. M. Jayarao. 2005. A survey on antibiotic usage in dairy herds in Pennsylvania. J. Dairy Sci. 88:2991-2999.

Selim, S. A., and J. S. Cullor. 1997. Number of viable bacteria and presumptive antibiotic residues in milk fed to calves on commercial dairies. J. Am. Vet. Med. Assoc. 211:1029-1035.

Smith, G. W., R. Gehring, A. L. Craigmill, A. I. Webb, and J. E. Riviere. 2005. Extralabel intramammary use of drugs in dairy cattle. J. Am. Vet. Med. Assoc. 226:1994-1996.

Thrusfield, M. 2005. Veterinary Epidemiology. 3rd ed. Butterworth, London, UK.

USDA. 2008a. Dairy 2007 Part I: Reference of Dairy Cattle Health and Management Practices in the United States. USDA, Animal and Plant Health Inspection Service (APHIS), Veterinary Services (VS), and National Animal Health Monitoring System (NAHMS), Fort Collins, $\mathrm{CO}$

USDA. 2008b. Antibiotic Use on U.S. Dairy Operations, 2002 and 2007. USDA, Animal and Plant Health Inspection Service (APHIS), Fort Collins, CO.
USDA. 2016. Dairy Cattle Management Practices in the United States, 2014. USDA, Animal and Plant Health Inspection Service (APHIS), Veterinary Services (VS), and National Animal Health Monitoring System (NAHMS), Fort Collins, CO.

Walz, P. H., T. P. Mullaney, J. A. Render, R. D. Walker, T. Mosser, and J. C. Baker. 1997. Otitis media in preweaned Holstein dairy calves in Michigan due to Mycoplasma bovis. J. Vet. Diagn. Invest. 9:250-254

WHO (World Health Organization). 2007. World Health Organization critically important antimicrobials for human medicine: Categorization for the development of risk management strategies to contain antimicrobial resistance due to non-human antimicrobial use. Report of the Second WHO Expert Meeting, Copenhagen, Denmark. WHO, Geneva, Switzerland. Accessed Jun. 6, 2018. http:// apps.who.int/iris/bitstream/handle/10665/43765/9789241595742 _eng.pdf;jsessionid=6E663E14D12000F53BCC6746BEA7399F ?sequence $=1$.

Zwald, A. G., P. L. Ruegg, J. B. Kaneene, L. D. Warnick, S. J. Wells, C. Fossler, and L. W. Halbert. 2004. Management practices and reported antimicrobial usage on conventional and organic dairy farms. J. Dairy Sci. 87:191-201. 